\title{
The Photodegradation of Ibuprofen and Dissolved Organic Matter in Lake Superior and St. Louis River Water
}

\author{
Angela B. Moynan and Cynthia A. Welsh
}

\begin{abstract}
Ibuprofen can enter bodies of water via waste water treatment. The question was what effect does photodegradation have on ibuprofen and dissolved organic matter (DOM) in Lake Superior (oligiotrophic) and St. Louis (tannic stained) River water? Ibuprofen concentrations of $15,000,30,000$, and $60,000 \mu \mathrm{g} / \mathrm{L}$ were made from lake, river, and distilled water, as well as additional distilled concentrations of 7,500 and 120,000 $\mu \mathrm{g} / \mathrm{L}$. Half of the eighty-four trial cups were placed in an ultraviolet light cabinet and half of the set were placed in a dark cabinet for three days. After the exposure period, a UV-Vis was performed to measure change in molar mass and the summed absorbance of colored dissolved organic matter (CDOM). It appears that ibuprofen decreases in molar mass after exposure to light in distilled and lake water with 15,000 $\mu \mathrm{g} / \mathrm{L}$ of ibuprofen. Surprisingly, the molar mass of DOM in river water increases after UV exposure. Possibly, this occurred because the river water has such a high molar mass of DOM and was not filtered. Microbial biomass could also have contributed to this increase. Ibuprofen entering bodies of water via the waste water treatment system appears to be affected by UV light exposure, but in different ways.
\end{abstract}

\section{Introduction}

D ISSOLVED ORGANIC MATTER (DOM) is a broad classification of organic molecules of varied origin and composition within aquatic systems. It links terrestrial and aquatic systems by providing nutrients for organisms. Dissolved organic carbon shields sunlight, affecting the competition between photosynthetic organisms, as well as contributing to the photochemical reactions within the water column ${ }^{1-3}$ Dissolved organic carbon is introduced to watersheds through either allochthonous (from outside the watershed) or autochthonous (from inside the watershed) inputs. ${ }^{4}$

There have been few studies done on large lakes such as Lake Superior (the world's largest freshwater lake by area) on a molecular level. Lake Superior, an oligotrophic lake, has very low concentrations of DOM. The St. Louis River, part of the Lake Superior watershed, flows through a large bog, and has much higher concentrations of DOM. Ibuprofen is considered a form of DOM when in an aquatic environment (C. Welsh, personal communication). ${ }^{1}$

There have been many instances in which pharmaceuticals, such as ibuprofen, have been identified in wastewater effluent. $^{5}$ Ibuprofen is a nonsteroidal anti-inflammatory drug (NSAID). ${ }^{6}$ In surface water, contaminants such as ibuprofen, if exposed to solar radiation, could possibly be photo- degraded, changing the chemical composition of the drugs, consequently affecting aquatic ecosystems. ${ }^{7}$ There are two ways that sunlight can alter DOM. The first is direct photolysis, which is the transformation of a compound due to its absorption of ultraviolet (UV) light. The energy from the UV light exposure interacts directly with the molecule, causing molecular vibration, leading to the breaking of bonds that may lead to free radical formation. The second way sunlight can alter DOM is indirectly. The directly photolysed free radical (photosensitizer or reactive oxygen species) can transform other DOM molecules, creating free radicals that will continue to react with other DOM molecules. ${ }^{8}$

Ibuprofen is a pharmaceutical that is continually being released into the environment through human activities. Schwaiger et al. ${ }^{9}$ found that long-term exposure of ibuprofen resulted in histopathological (microscopic anatomical changes in diseased tissue) changes in the gills and kidneys of fish. Also, in mammals, NSAIDs have been shown to alter ovulation and development. ${ }^{10,11}$ Ibuprofen can be considered an endocrine disruptor when absorbed into the body. Endocrine disruptors mimic or block hormones and disrupt the body's normal functions. ${ }^{12}$ With increasing concentrations of ibuprofen, Japanese Medaka fish were shown to spawn less frequently and produce more eggs when they did spawn. ${ }^{9,12}$ Very few studies have been done on large lakes, such as Lake Superior, at a molecular level, therefore, understanding

Cloquet Senior High School, Cloquet, Minnesota. 
the direct and indirect way sunlight affects ibuprofen molecules entering aquatic systems via waste water effluent is important.

This led to the following question and hypotheses; the question was: what effect does photodegradation have on ibuprofen and dissolved organic matter in Lake Superior (oligotrophic) and St. Louis River (tannic stained) water? The first hypothesis was: if ibuprofen and DOM are photodegradated in Lake Superior and St. Louis River water, then the molar mass of the DOM will be reduced. The second hypothesis was: if ibuprofen concentrations are photodegraded, then the summed absorbance of the CDOM in the water will be reduced. Also, the summed absorbance of the CDOM will be higher in the river water than in the lake water.

\section{Methods}

\section{Initial toxicity experiments}

An experiment was done in 2009 to determine the toxicity of different ibuprofen concentrations in Lake Superior (oligotrophic) and St. Louis River (tannic stained) water before and after exposure to UV light on Daphnia magna. River water both with and without ibuprofen enhanced Daphnia magna's survival, yet did not affect reproduction. Lake water with ibuprofen exposed to UV light had a negative affect on Daphnia magna survival, but a positive affect on reproduction (Table 1$)^{2}$

A study done in 2010 examined the toxicity of different ibuprofen concentrations $(15,000,30,000$, and 60,000 $\mu \mathrm{g} / \mathrm{L})$ in Lake Superior (oligotrophic) water and St. Louis River (tannic stained) water before and after exposure to UV light on an aquatic surface residing plant, Lemna minor, as well as a water column inhabiting green algae Selenastrum capricornutum. This study showed that Lemna minor's survival was positively affected in the Lake Superior water in the light. However, there was no significant difference in the chlorophyll- $a$ absorbance of Selenastrum capricornutum when compared to all the treatments in both Lake Superior and St. Louis River water (see Table 1). ${ }^{3}$ Both studies indicate that ibuprofen is having an effect on aquatic organisms, especially in the oligotrophic lake water (Lake Superior) with ibuprofen after light exposure. This led to this year's question: what is happening to ibuprofen, a form of dissolved carbon (DOC), at the molecular level in both Lake Superior (oligotrophic) and St. Louis River (tannic stained) water before and after exposure to UV light?

This year's study was done to determine what is happening to ibuprofen, a form of DOC, at the molecular level in both Lake Superior (oligotrophic) and St. Louis River (tannic stained) water before and after exposure to UV light. Filtered Lake Superior (oligotrophic) water was obtained from the Environmental Protection Agency (EPA) in Duluth, Minne-

Table 1. Treatment Comparisons to the Control SAMPLE Without IBUPROFEN ${ }^{2,3}$

\begin{tabular}{lcccc}
\hline & $\begin{array}{c}\text { Daphnia } \\
\text { magna } \\
\text { survival }\end{array}$ & $\begin{array}{c}\text { Daphnia } \\
\text { magna } \\
\text { reproduction }\end{array}$ & $\begin{array}{c}\text { Algal } \\
\text { growth }\end{array}$ & $\begin{array}{c}\text { Frond } \\
\text { growth }\end{array}$ \\
\hline Lake light & - & + & None & + \\
River light & + & None & None & None \\
\hline
\end{tabular}

sota (Fig. 1). Unfiltered St. Louis River (tannic stained) water was obtained at Safford Park in Cloquet, Minnesota. The previous study done in 2010 examined the toxicity at ibuprofen concentrations of $15,000,30,000$, and $60,000 \mu \mathrm{g} / \mathrm{L}$; this year's study added two additional concentrations (7500 and $120,000 \mu \mathrm{g} / \mathrm{L})$. Dilutions were made by crushing up a 200 milligram (mg) tablet of over-the-counter ibuprofen with a mortar and pestle. Ibuprofen was then mixed with 200,000 milliliters $(\mathrm{mL})$ of lake water to create a concentration of 200,000 micrograms per liter $(\mu \mathrm{g} / \mathrm{L})$. The $200,000 \mu \mathrm{g} / \mathrm{L}$ concentration was then serial diluted into concentrations of $120,000 \mu \mathrm{g} / \mathrm{L}$ and $100,000 \mu \mathrm{g} / \mathrm{L}$. Additional concentrations were then diluted from the $100,000 \mu \mathrm{g} / \mathrm{L}$ into $60,000 \mu \mathrm{g} / \mathrm{L}$, $30,000 \mu \mathrm{g} / \mathrm{L}, 15,000 \mu \mathrm{g} / \mathrm{L}$ and 7,500 $\mu \mathrm{g} / \mathrm{L}$. The above procedure was repeated with St. Louis River (tannic stained) and distilled water.

Three sets of 18 plastic containers were filled with $500 \mathrm{~mL}$ of each of the dilutions. One set with distilled water, one set with Lake Superior (oligotrophic) water, and one set with St. Louis River (tannic stained) water were placed into an ultraviolet B (254.7 nanometers) sanitizing monitor cabinet. After locking the cabinet, the light was turned on and left in the cabinet for 3 days, then removed. Albaigés et al. ${ }^{7}$ found that ibuprofen photodegraded at a half-life of $1-5 \mathrm{~h}$, while Cleuvers ${ }^{13}$ found a change in Daphnia mobility after ibuprofen was photodegraded for $48 \mathrm{~h}$. After discussing these time frames with an EPA toxicologist, it was determined that ibuprofen samples in Lake Superior and St. Louis River water would be exposed to UV light for 3 days. Another set of 18 plastic containers filled with $500 \mathrm{~mL}$ of each of the dilutions, one set with Lake Superior (oligotrophic) water, and one set with St. Louis River (tannic stained), and distilled water were placed in a dark cabinet. After the 3 day exposure period, all of the concentrations were removed from their cabinets and transported to the University of Minnesota Duluth's Large Lakes Observatory to perform a UV-Vis spectrophotometer on the samples.

The Large Lake Observatory Standard Operating Procedures (SOP) for the UV-Vis spectrophotometer were used to determine the molar mass of DOM using the $\mathrm{e} 2 / \mathrm{e} 3$ ratio. The e2/e3 ratio is read at 250 nanometers $(\mathrm{nm})(\mathrm{e} 2)$ and $365 \mathrm{~nm}$ (e3), the ratio is inversely proportional to the molar mass. Also, a comparison of CDOM, which is the sum of the normalized absorption coefficients from $250 \mathrm{~nm}$ to $400 \mathrm{~nm}$, was done using the Genesis $6 \mathrm{UV}$-Vis spectrophotometer. A higher

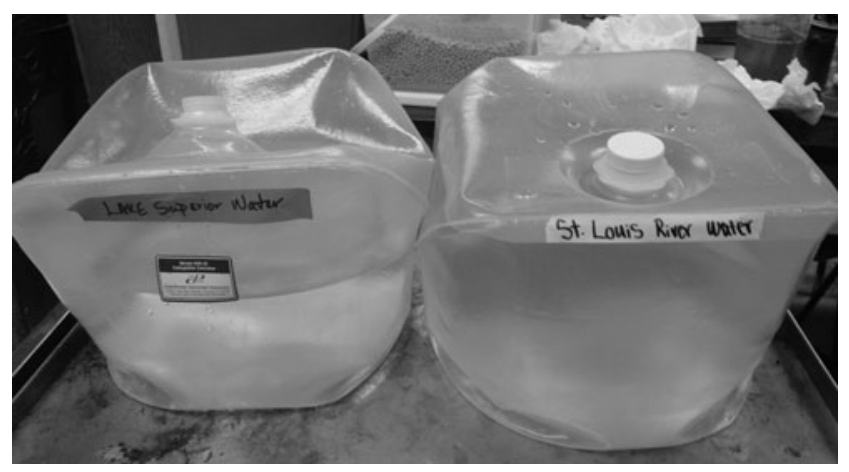

FIG. 1. The St. Louis River and Lake Superior water used in ibuprofen dilutions exposed to UV light. 
absorption coefficient reading indicates an elevated concentration of CDOM over other samples.

To begin using the UV-Vis spectrophotometer, a quartz cuvette was rinsed 3 times with milliQ water (MQW) and wiped well with a carbon free, nonscratching lab wipe. A baseline test was run. The baseline was left in the UV-Vis spectrophotometer and a new cuvette was cleaned 3 times with MQW, and 3 times with the sample. The cuvette was wiped well each time and then analyzed by the UV-Vis spectrophotometer. Blanks were run at the beginning of the testing period, at the end of the testing, as well as periodically throughout the testing (Fig. 2). CDOM and e2/e3 analysis of UV-Vis spectrophotometer data was done using a spreadsheet. The data was adjusted for blanks and backscatter to determine the absorption coefficient.

In order to determine if there was a significant difference in molar mass and CDOM because of UV light exposure, water type and/or ibuprofen concentration, a univariate analysis of variance (ANOVA) was done. Significance was determined when the probability or $p$ value was $p<0.05$. This level indicates that the differences observed were due to the treatment not just due to chance.

\section{Results}

Figure 3 shows the e2/e3 ratio using a UV-Vis spectrophotometer read at 250 nanometers $(\mathrm{nm})(\mathrm{e} 2)$ and $365 \mathrm{~nm}(\mathrm{e} 3)$. In distilled water exposed to UV light, ibuprofen concentrations of 7,500, 15,000, 60,000, and 120,000 $\mu \mathrm{g} / \mathrm{L}$ had a significantly higher $\mathrm{e} 2 / \mathrm{e} 3$ ratio (indicating smaller molar mass) than all nonlight-treated ibuprofen samples (indicating a reduction in molar mass), ANOVA $p<0.001$.

Figures 4 and 5 show the e2/e3 ratios for the St. Louis River and Lake Superior samples, respectively. In river water exposed to UV light, ibuprofen concentrations of $15,000,30,000$, and $60,000 \mu \mathrm{g} / \mathrm{L}$, as well as the control group exposed to the light, had a significantly larger molar mass when compared to the ibuprofen concentrations in the dark, with a $p$ value of $<0.021$. In the lake water exposed to UV light, and not exposed to UV light, the ibuprofen concentrations of 30,000, and

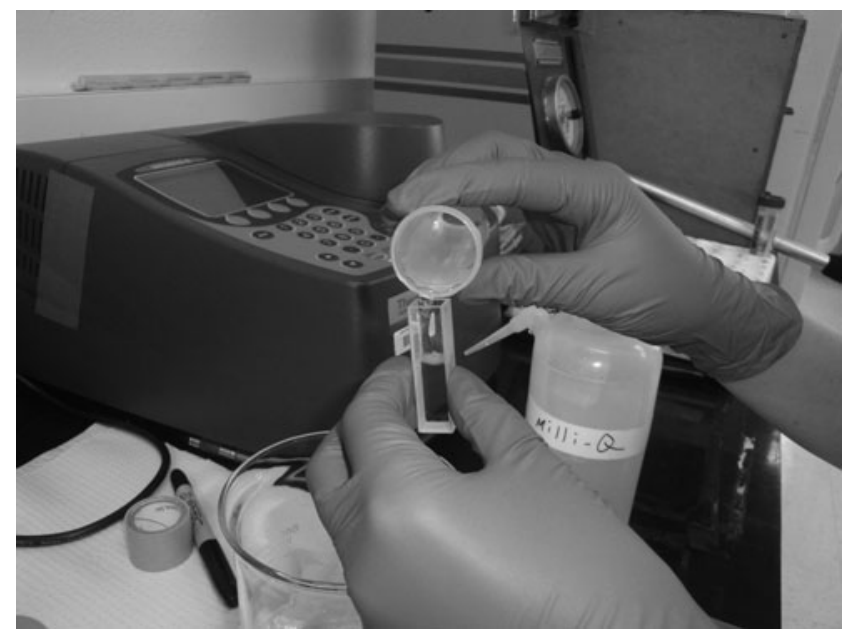

FIG. 2. Pouring a St. Louis River water sample into a cuvette, in order to be read by a UV-Vis Spectrophotometer at UMD's Large Lake Observatory in Duluth, MN.

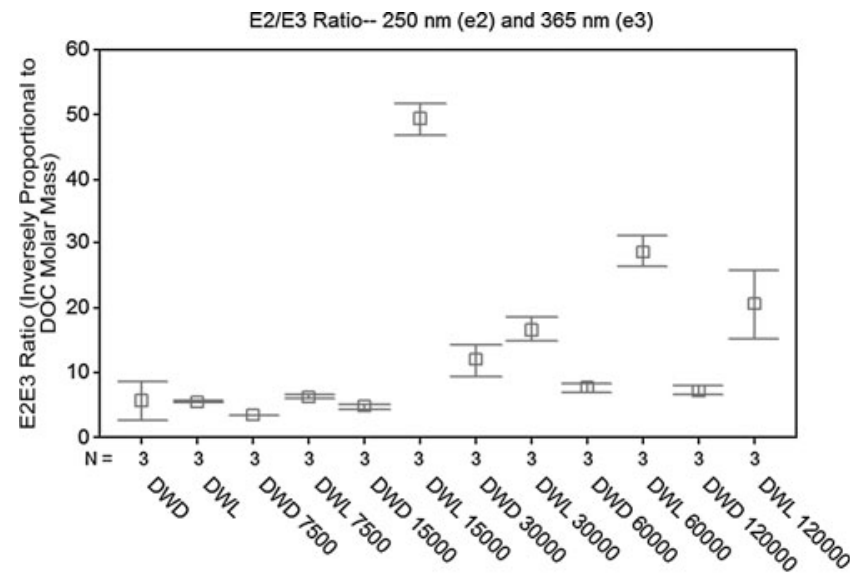

Distilled Water lbuprofin ANOVA DWD/DWL p<.001, DWD IBP DWL (15/60/120)

FIG. 3. Using a UV-Vis on distilled water.

$60,000 \mu \mathrm{g} / \mathrm{L}$, as well as the controls for both samples, were not significantly different in molar mass (size of DOM). There was a significantly lower molar mass for the $15,000 \mu \mathrm{g} / \mathrm{L}$ samples exposed to light when compared to no light exposure as the concentrations increased, using an ANOVA with a $p$ value of $<0.001$.

Figure 6 shows a comparison of river water and lake water concentrations of ibuprofen when exposed to UV light using the e2/e3 ratio from a UV-Vis spectrophotometer read at $250 \mathrm{~nm}(\mathrm{e} 2)$ and $365 \mathrm{~nm}$ (e3). When compared to the control and all other concentrations, river water's molar mass of DOM was significantly larger than the molar mass of the lake water, using an ANOVA with a $p$ value of $<0.0001$. When the lake water control was exposed to UV light, there was a significant reduction in molar mass. Also, when the lake water control exposed to light was compared to the lake water with ibuprofen exposed to light, the ibuprofen samples had an overall significant reduction in molar mass.

Figures 7 and 8 show the CDOM in summed absorbance using a UV-Vis spectrophotometer. In the lake water treated to ultraviolet (UV) light, ibuprofen concentrations of 15,000, 30,000 , and $60,000 \mu \mathrm{g} / \mathrm{L}$, as well as the control for both samples exposed to UV light, and those that were not, had a significant reduction in the summed absorbance of CDOM in the

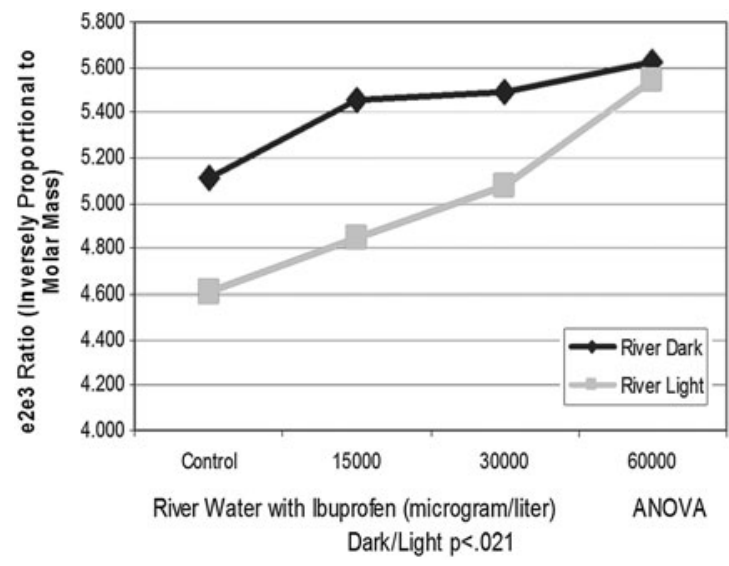

FIG. 4. River water: using a UV-Vis, e2e3 ratio $250 \mathrm{~nm}(\mathrm{e} 2)$ and $365 \mathrm{~nm}(\mathrm{e} 3)$. 


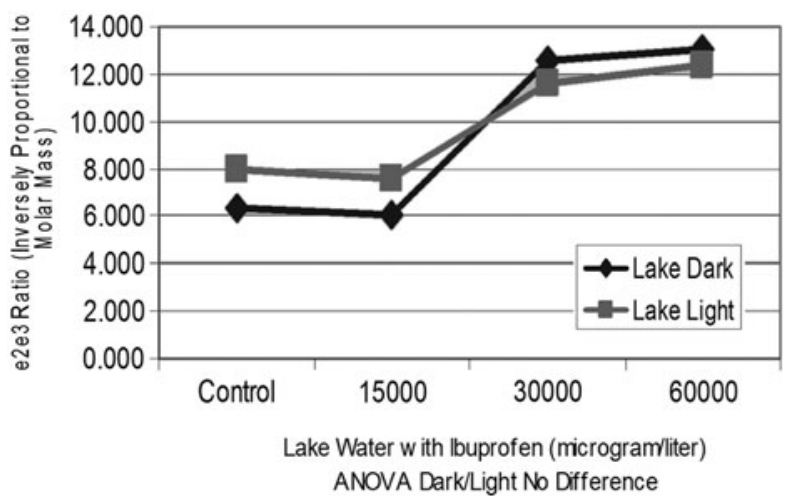

FIG. 5. Lake water: using a UV-Vis, e2e3 ratio $250 \mathrm{~nm}(\mathrm{e} 2)$ and $365 \mathrm{~nm}(\mathrm{e} 3)$.

lake water for all ibuprofen concentrations exposed to light when compared to the dark-treated water, using an ANOVA with a $p$ value of $<0.027$. In river water treated to UV light, ibuprofen concentrations of 15,000, 30,000, and 60,000 $\mu \mathrm{g} / \mathrm{L}$, as well as the controls for both samples exposed to UV light, and those that were not, showed no significant difference in the summed absorbance of CDOM. In the lake water for all ibuprofen concentrations exposed to UV light, when compared to the dark-treated water, there was a significant decrease in CDOM when samples were exposed to UV light, using an ANOVA with a $p$ value of $<0.027$.

Figure 9 shows a comparison of CDOM in summed absorbance using a UV-Vis spectrophotometer for river water and lake water concentrations of ibuprofen when exposed to UV light. When compared to lake water, the river water's summed absorbance was significantly higher than the lake water, using an ANOVA with a $p$ value of $<0.001$.

\section{Conclusion/Discussion}

The original hypothesis was: if ibuprofen concentrations in distilled, Lake Superior and St. Louis River water were photodegradated, then the molar mass (size) of DOM would be reduced. The first hypothesis was supported when distilled water concentrations of ibuprofen were exposed to UV light for all concentrations. However, there was no difference when

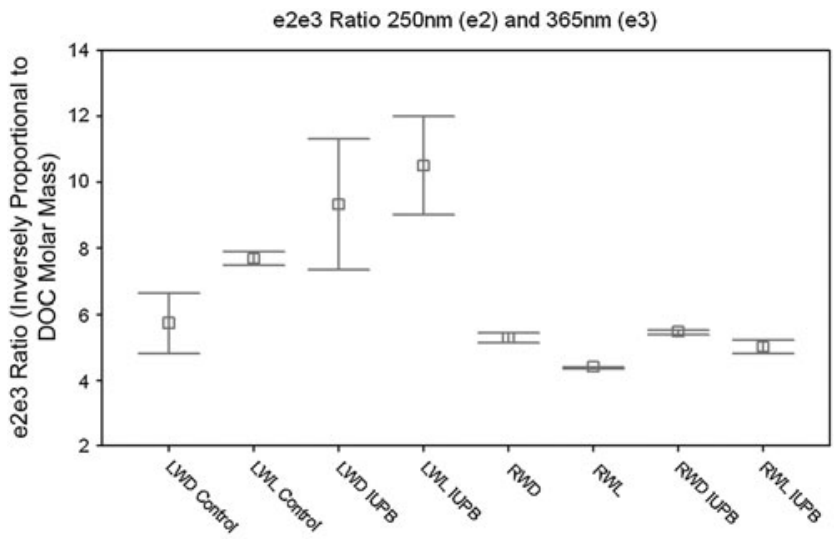

Lake/River Ibup: ANOVA RWD/RWL p<.021; LWD/LWL no diff; Water $p<.0001$

FIG. 6. Using a UV-Vis on river and lake water, e2e3 ratio $250 \mathrm{~nm}(\mathrm{e} 2)$ and $365 \mathrm{~nm}(\mathrm{e} 3)$.

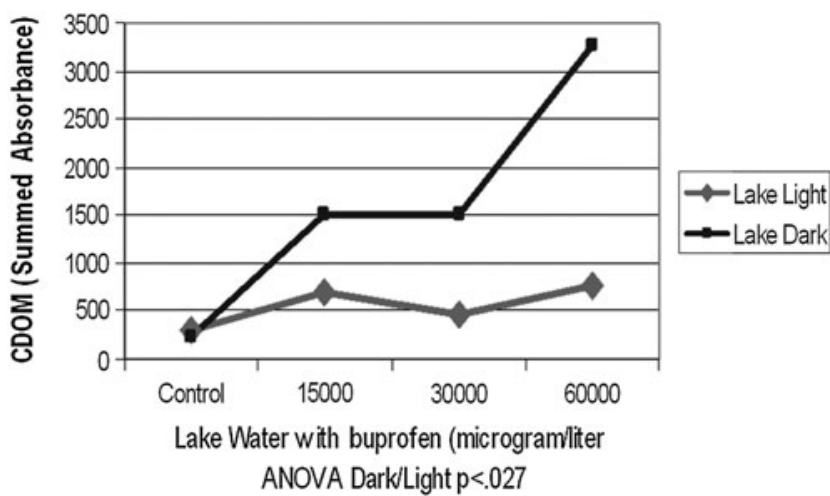

FIG. 7. Lake water: using a UV-Vis colored dissolved organic matter (CDOM).

lake water was exposed to light for the higher concentrations of ibuprofen (30,000 and 60,000 $\mu \mathrm{g} / \mathrm{L})$. This may have been due to undissolved particles of ibuprofen. When the samples with $15,000 \mu \mathrm{g} / \mathrm{L}$ of ibuprofen dissolved in lake water, and the control without ibuprofen were both exposed to UV light, molar mass significantly decreased. It appears that the Lake Superior water control and the samples with $15,000 \mu \mathrm{g} / \mathrm{L}$ of ibuprofen, after exposure to UV light, photodegradated in the same manner as the distilled water samples with ibuprofen. These results possibly indicate that the DOM in Lake Superior is similar in structure (molar mass) to the structure (molar mass) of ibuprofen. It appears that ibuprofen decreases in molar mass after exposure to light in distilled water, and at lower concentrations in lake water $(15,000 \mu \mathrm{g} / \mathrm{L})$ due to direct photolysis, or transformation due to its absorption of UV light. Surprisingly, the ibuprofen samples in river water showed a significant increase in molar mass after exposure to UV light. This may have occurred because the river water had such a high molar mass of DOM to begin with and the water was not filtered. Microbial biomass could also have contributed to this increase.

The second hypothesis was: if ibuprofen concentrations were photodegraded, then the summed absorbance of the CDOM in the water would be reduced. This hypothesis was partially supported. In the lake water concentrations, the summed absorbance of CDOM was significantly reduced after exposure to UV light. The river water ibuprofen concentrations had a summed absorbance of CDOM that was significantly higher after exposure to UV light. This may

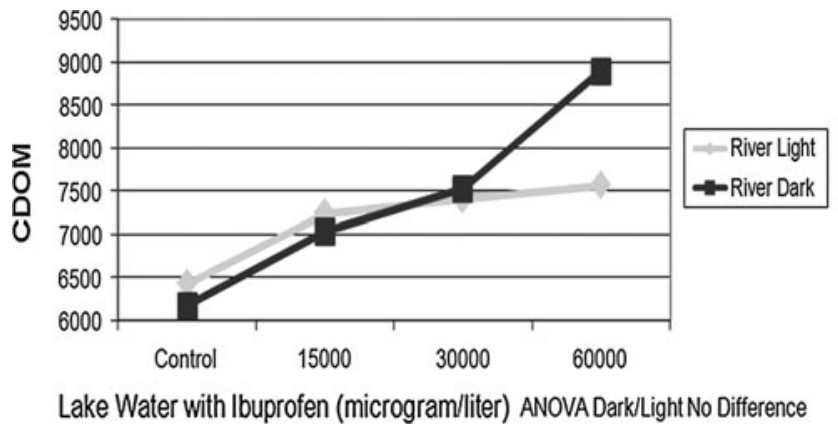

FIG. 8. River water: using a UV-Vis colored dissolved organic matter (CDOM). 


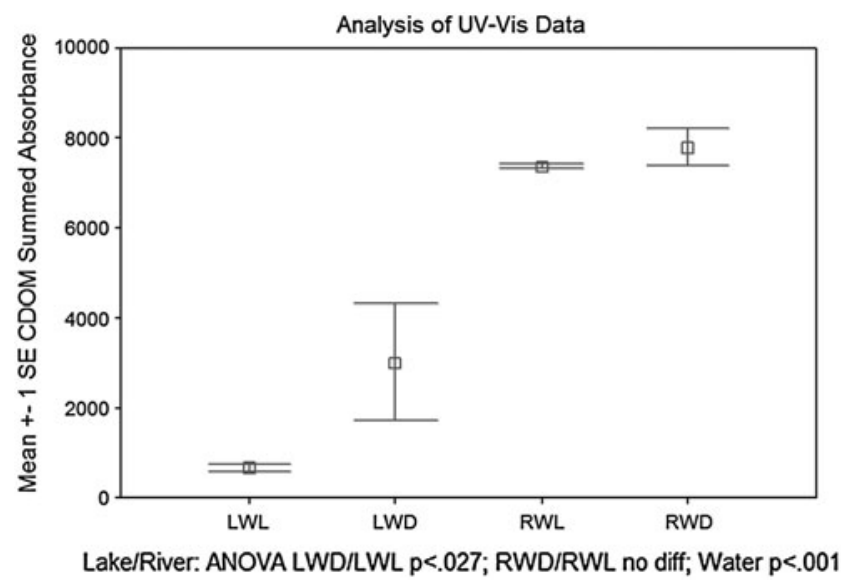

FIG. 9. Colored dissolved organic matter (CDOM).

have occurred because the initial river water itself had such a high molar mass of DOM. Also, the lake water was filtered by the Environmental Protection Agency, but the St. Louis River water was collected directly from the river and was not filtered. Microbial biomass may have contributed to this increase. The hypothesis that the summed absorbance of CDOM would be higher in the river water than in the lake water was also supported. The brown river water appeared to be tannic (organic) stained, because the UV-Vis spectrophotometer indicated a significantly higher CDOM. When the river water was compared to lake water, it appears the brown color indicates the presence of CDOM.

Duke and Moynan ${ }^{2}$ found that Lemna minor growth was positively affected by the presence of ibuprofen in lake water treated to UV light, but not in river water. This year's study indicates that the molar mass of DOM in Lake Superior water decreased after exposure to UV light. Possibly, the photolysis of DOM may be making dissolved ibuprofen (DOM) more bioavailable, or possibly less toxic for Lemna minor. Duke and Moynan ${ }^{2}$ found that Daphnia magna's survival was negatively affected in lake water with dissolved ibuprofen treated to UV light, while reproduction was enhanced. Possibly, as ibuprofen photodegrades, as this year's study indicates, the resulting chemicals may be more toxic to the adult, thus decreasing survival, while surprisingly increasing the reproduction rate of the remaining adults.

These results are similar to what Flippin et al. ${ }^{12}$ found when studying Japanese Medaka fish. With increasing concentrations of ibuprofen, Japanese Medaka fish were shown to spawn less frequently and produce more eggs when they did spawn. Thus, photodegraded ibuprofen may act as an endocrine disrupter. It appears that ibuprofen molecules entering aquatic systems via waste water effluent are being photodegraded after exposure to direct and indirect sunlight, altering aquatic systems in various ways. Continued research needs to be done on large lakes, such as Lake Superior, at the molecular level.

\section{Acknowledgments}

The professional mentor in the initial phase of this research (2009) was Dr. Dave Mount from the Environmental Protection Agency in Duluth, Minnesota. Dr. Mount sup- plied Lake Superior water and gave instruction on how to make serial dilutions. This year, Elizabeth Welsh, a graduate student at the Large Lakes Observatory in Duluth, Minnesota provided professional guidance. Elizabeth works under Dr. Elizabeth Minor, a chemical limnology professor at the University of Minnesota Duluth. Dr. Minor provided work space at the Large Lakes Observatory. Welsh provided training in laboratory safety and procedures. Elizabeth Welsh also edited initial versions of this article and provided guidance in data analysis. Melissa Olson, a high school English teacher at Prior Lake High School-Bridges Area Learning Center, in Prior Lake, Minnesota, performed the final edit before submission. Special thanks goes to Marlena Duke, a Cloquet High School senior, for her contributions in the initial phase of this work.

\section{Author Contributions}

Angela B. Moynan is a high school student at Cloquet Senior High School in Cloquet, MN. Angela performed this research as a part of her 2011-2012 Science Fair project. She presented her work at several conferences including the MN Academy of Science State Science Fair and the Tri-State Junior Science and Humanities Symposium. Her research was selected as one of the top water research papers for the $2012 \mathrm{MN}$ Stockholm Junior Waterprize and she was selected to attend the Intel International Science and Engineering Fair in Pittsburgh, PA.

Cynthia A. Welsh has a doctorate in Educational Policy and Administration from the University of Minnesota and is currently a science teacher at both Cloquet Senior High and Middle School. She is also the director of the NE Minnesota and American Indian Science and Engineering Regional Fair. She co-mentored Angela Moynan in her DOM research, as well as provided training, technical support, and guidance throughout the research and writing process.

\section{Disclosure Statement}

No competing financial interests exist.

\section{References}

1. Minor E, Stephens B. Dissolved organic matter characteristics within the Lake Superior watershed. Org Geochem 2008;39:1498-1501.

2. Duke M, Moynan A. Phantom pharmaceuticals: What effect does ibuprofen treated with ultraviolet light in clear lake water (Lake Superior) and tannic stained river water (St. Louis River) have on Daphnia magna's reproduction and survival: Phase II? Tri-State Junior Science and Humanities Symposium, Bloomington, MN, 2010.

3. Duke M, Moynan A. Phantom pharmaceuticals: What effect does ibuprofen treated with ultraviolet light in clear lake water (Lake Superior) and tannic stained river water (St. Louis River) have on Selenastrum capricornutum and Lemna minor reproduction and survival: Phase III? Tri-State Junior Science and Humanities Symposium, Bloomington, MN, 2011.

4. Minor E, Simjouw J, Mulholland M. Seasonal variations in dissolved organic carbon concentrations and characteristics in a costal bay. Marine Chem 2006;101:166-179.

5. Zuehlke S, Duennbier U, Heberer T. Investigation of the behavior and metabolism of pharmaceutical residues during 
purification of contaminated ground water used for drinking supply. Chemosphere 2007;1673-1680.

6. Cerner Multum Inc. Available at www.drugs.com $/ \mathrm{mtm} /$ ibuprofen.html\#, accessed September 25, 2008.

7. Matamoros V, Duhec A, Albaigés J, Bayona J. Photodegradation of carbamazepine, ibuprofen, ketoprofen and $17 \alpha$-ethinylestradiol in fresh and sea water. Water Air Soil 2008;161-168.

8. Rodenburg LA. Photochemical transformation reactions. John Hopkins University: Environmental Organic Chemistry Class Presentation, 2011.

9. Schwaiger J, Ferling H, Mallow U, Wintermayr H, Negele RD. Toxic effects of the non-steroidal anti-inflammatory drug diclofenac: Part I: Histopathological alterations and bioaccumulation in rainbow trout. Aquatic Toxicol 2004;141-150.

10. Triebskorn R, Casper H, Heyd A, Eikemper R, Köhler HR, Schwaiger J. Toxic effects of the non-steroidal antiinflammatory drug diclofenac: Part II: Cytological effects in liver, kidney, gills, and intestine of rainbow trout (Oncorhynchus mykiss). Aquatic Toxicol 2004;151-166.
11. Fatoki O, Opeolu B. Studies on the occurrence and quantification of phenolic endocrine disruptors in water. Sci Res Essay 2009;1415-1422.

12. Flippin JL, Huggett D, Foran CM. Changes in the timing of reproduction following chronic exposure to ibuprofen in Japanese medake, Oryzias latipes. Aquatic Toxicol 2006; 73-78.

13. Cleuvers M. Mixture toxicity of the anti-inflammatory drugs diclofenac, ibuprofen, naproxen, and acetylsalicylic acid. Ectoxicol Environ Safety 2003;59: 309-315.

Address correspondence to: Cynthia A. Welsh, B.S., M.Ed., Ed.D. Cloquet Senior High School $100018^{\text {th }}$ Street Cloquet, MN 55720

E-mail: cwelsh@cloquet.k12.mn.us 